\title{
On a Generic Dimension of the Critical Locus
}

\author{
Grzegorz Oleksik@
}

\begin{abstract}
Let $f:\left(\mathbb{C}^{n}, 0\right) \rightarrow(\mathbb{C}, 0), n \leq 3$, be a nondegenerate singularity. In this article we give a combinatorial characterization of the dimension of the critical locus of $f$ in terms of its support. We also show that this dimension can be read off from the Newton diagram of $f$, which solves one of Arnold's problems in this case.
\end{abstract}

Mathematics Subject Classification. 32S05.

Keywords. Dimension, critical locus, singularity, Newton diagram, nondegeneracy, support.

\section{Introduction}

In 1968 and 1975 Vladimir I. Arnold posed the following problems (see [1]):

1968-2 What topological characteristics of a real (complex) polynomial are computable from the Newton diagram (and the signs of the coefficients)?

1975-1 Every interesting discrete invariant of a generic singularity with a Newton polyhedron $\Gamma$ is an interesting function of the polyhedron. Study: the signature, the number of moduli, the singularity index, the integral monodromy, the variation, the Bernstein polynomial, and $\mu_{i}$ (for generic section).

1975-21 Express the main numerical invariants of a typical singularity with a given Newton diagram (e.g., the signature, the genus of the 1-dimensional Milnor fiber) in terms of the diagram. 
Let $f:\left(\mathbb{C}^{n}, 0\right) \rightarrow(\mathbb{C}, 0)$ be a nondegenerate singularity (see Sect. 2$)$. The dimension $d$ of the critical locus of $f$ is a discrete invariant of a singularity. The purpose of this paper is to partially solve the above Arnold's problems for $d$ in the case $n \leq 3$. Precisely we show that $d$ depends only on the Newton diagram of $f$ (see Theorem 3.3). Moreover this dimension can be easily read off from the Newton diagram by checking some combinatorial condition called the (d)-Kouchnirenko condition (see Sect. 2 and Theorem 3.4). Firstly we show that $d$ is determined by the support of $f$ (see Theorem 3.2). Then we deduce Theorems 3.3 and 3.4 from this fact. We also give a simple characterization of a nondegenerate singularity, when its critical locus has codimension one, for arbitrary $n$ (see Propositions 4.5 and 4.6). As an application of Theorem 3.2 we give Corollaries 4.8 and 4.9.

Kouchnirenko in $\left[6\right.$, Thm 1] gave for a set $M \subset \mathbb{N}^{n}$ a necessary and sufficient condition (called in [2] the Kouchnirenko condition) so that there exists an isolated singularity $f$ with supp $f \subset M$. In the joint paper [2] (for arbitrary $n$ ) it is proved that the fulfillment of the Kouchnirenko condition by the support of a nondegenerate singularity $f$ is equivalent to $f$ being an isolated singularity. There are some equivalent combinatorial conditions to the Kouchnirenko condition. Hertling and Kurbel collected such conditions for quasihomogeneous polynomial in [4, Lemma 2.1] but this lemma is also true without the assumption of quasihomogeneity. On the other hand, Kouchnirenko writes in Remark 1.13 (ii) of his celebrated paper [5] that the Newton number of a singularity $f$ is finite if and only if supp $f$ satisfies the Kouchnirenko condition.

The $(d)$-Kouchnirenko condition is a generalization of the Kouchnirenko condition. The (0)-Kouchnirenko condition is exactly the Kouchnirenko condition (see Sect. 2). When $n \leq 3$ Theorem 3.1 (the main result of [2]) is a special case of Theorem 3.2 for $d=0$.

\section{Preliminaries}

Let $f:\left(\mathbb{C}^{n}, 0\right) \longrightarrow(\mathbb{C}, 0)$ be a nonzero holomorphic function in an open neighborhood of $0 \in \mathbb{C}^{n}$. We say that $f$ is a singularity if $f(0)=0, \operatorname{grad} f(0)=$ 0 , where $\operatorname{grad} f=\left(f_{z_{1}}^{\prime}, \ldots, f_{z_{n}}^{\prime}\right)$. We say that $f$ is an isolated singularity if $f$ is a singularity, which has an isolated critical point in the origin i.e. additionally $\operatorname{grad} f(z) \neq 0$ for $z \neq 0$ near 0 . We note $\mathbb{N}=\{0,1,2, \ldots\}$. Let $\sum_{\nu \in \mathbb{N}^{n}} a_{\nu} z^{\nu}$ be the Taylor expansion of $f$ at 0 . We define the set $\operatorname{supp} f=\left\{\nu \in \mathbb{N}^{n}: a_{\nu} \neq 0\right\}$ and call it the support of $f$. We define

$$
\Gamma_{+}(f)=\operatorname{conv}\left\{\nu+\mathbb{R}_{+}^{n}: \nu \in \operatorname{supp} f\right\} \subset \mathbb{R}^{n}
$$

and call it the Newton diagram of $f$. Let $u \in \mathbb{R}_{+}^{n} \backslash\{0\}$. Put

$$
\begin{aligned}
l\left(u, \Gamma_{+}(f)\right) & =\inf \left\{\langle u, v\rangle: v \in \Gamma_{+}(f)\right\}, \\
\Delta\left(u, \Gamma_{+}(f)\right) & =\left\{v \in \Gamma_{+}(f):\langle u, v\rangle=l\left(u, \Gamma_{+}(f)\right)\right\} .
\end{aligned}
$$


We say that $S \subset \mathbb{R}^{n}$ is a face of $\Gamma_{+}(f)$ if $S=\Delta\left(u, \Gamma_{+}(f)\right)$ for some $u \in$ $\mathbb{R}_{+}^{n} \backslash\{0\}$. The vector $u$ is called a primitive vector of $S$. It is easy to see that $S$ is a closed and convex set and $S \subset \operatorname{Fr}\left(\Gamma_{+}(f)\right)$, where $\operatorname{Fr}(A)$ denotes the boundary of $A$. One can prove that a face $S \subset \Gamma_{+}(f)$ is compact if and only if all coordinates of its primitive vector $u$ are positive. We call the family of all compact faces of $\Gamma_{+}(f)$ the Newton boundary of $f$ and denote it by $\Gamma(f)$. For every compact face $S \in \Gamma(f)$ we define the quasihomogeneous polynomial $f_{S}=\sum_{\nu \in S} a_{\nu} z^{\nu}$. We say that $f$ is nondegenerate on the face $S \in \Gamma(f)$ if the system of equations

$$
\frac{\partial f_{S}}{\partial z_{1}}=\ldots=\frac{\partial f_{S}}{\partial z_{n}}=0
$$

has no solution in $\left(\mathbb{C}^{*}\right)^{n}$, where $\mathbb{C}^{*}=\mathbb{C} \backslash\{0\}$. We say that $f$ is nondegenerate in the sense of Kouchnirenko (in short nondegenerate) if it is nondegenerate on each face of $\Gamma(f)$.

Let $M \subset \mathbb{N}^{n}$. Define the sets $M_{i}=\left\{\nu \in \mathbb{N}^{n}: \nu+e_{i} \in M\right\}$, where $e_{i}, i=$ $1, \ldots, n$, is the standard basis in $\mathbb{R}^{n}$. Notice that if we take $f_{M}=\sum_{m \in M} z^{m}$ then $M_{i}=\operatorname{supp} \partial f_{M} / \partial z_{i}$ for every $i=1,2, \ldots, n$. Let $I \subset\{1, \ldots, n\}$. Set

$$
O X_{I}=\left\{x \in \mathbb{R}^{n}: x_{i}=0, i \notin I\right\} .
$$

Observe that $O X_{I}$ is the hyperplane spanned by axes $O X_{i}, i \in I$.

Let $I \subset\{1,2, \ldots, n\}, d \in \mathbb{N}, 0 \leq d \leq n$.

Definition 2.1. We say that $M$ satisfies the $(d)$-Kouchnirenko condition for $I$ if there exist at least $|I|-d$ nonempty sets among the sets $M_{1} \cap O X_{I}, \ldots, M_{n} \cap$ $O X_{I}$.

Definition 2.2. We say that $M$ satisfies the $(d)$-Kouchnirenko condition if $M$ satisfies the $(d)$-Kouchnirenko condition for every $I \subset\{1,2, \ldots, n\}$.

If $d=0$ instead of the (0)-Kouchnirenko condition we will write simply the Kouchnirenko condition.

Remark 2.3. It is easy to check that $M$ satisfies the $(d)$-Kouchnirenko condition if and only if a finite subset of $M$ satisfies the $(d)$-Kouchnirenko condition.

\section{Main Results}

In this section we give the main results of this paper. The following result was proved in [2].

Theorem 3.1. Let $f:\left(\mathbb{C}^{n}, 0\right) \rightarrow(\mathbb{C}, 0)$ be a nondegenerate singularity. The following conditions are equivalent.

(i) $\operatorname{dim}_{0} \Sigma f=0$,

(ii) $\operatorname{supp} f$ satisfies the Kouchnirenko condition 
The aim of this article is to move the above theorem to the case of a non-isolated singularity. Precisely we show that the dimension of the critical locus of a nondegenerate singularity is determined by its support in the case $n \leq 3$. To compute this dimension it is enough to check simple combinatorial conditions imposed on the support.

Let $n \leq 3$.

Theorem 3.2. Let $f:\left(\mathbb{C}^{n}, 0\right) \rightarrow(\mathbb{C}, 0)$ be a nondegenerate singularity. The following conditions are equivalent.

(i) $\operatorname{dim}_{0} \Sigma f=d$,

(ii) supp $f$ satisfies the $(d)$-Kouchnirenko condition and does not satisfy the $(d-1)$-Kouchnirenko condition,

$0 \leq d \leq n$.

The second result shows that the dimension of the critical locus of a nondegenerate singularity depends only on its Newton diagram.

Theorem 3.3. Let $f, g:\left(\mathbb{C}^{n}, 0\right) \rightarrow(\mathbb{C}, 0)$ be nondegenerate singularities. If $\Gamma_{+}(f)=\Gamma_{+}(g)$, then $\operatorname{dim}_{0} \Sigma f=\operatorname{dim}_{0} \Sigma g$.

As a direct consequence of Theorems 3.2 and 3.3 we get the following.

Theorem 3.4. Let $f:\left(\mathbb{C}^{n}, 0\right) \rightarrow(\mathbb{C}, 0)$ be a nondegenerate singularity. Let $V$ be the set of vertices of $\Gamma_{+}(f)$. The following conditions are equivalent.

(i) $\operatorname{dim}_{0} \Sigma f=d$,

(ii) $V$ satisfies the $(d)$-Kouchnirenko condition and does not satisfy the $(d-$ 1) -Kouchnirenko condition,

$0 \leq d \leq n$.

This last result show that the dimension of the critical locus of a nondegenerate singularity can be read off from the Newton diagram of $f$. To compute this dimension it is enough to check the $(d)$-Kouchnirenko condition only for vertices of the Newton diagram of $f$.

\section{Proof of the Main Results}

We start with the following.

Proposition 4.1. Let $f:\left(\mathbb{C}^{n}, 0\right) \rightarrow(\mathbb{C}, 0), n \geq 2$, be a singularity. If $\operatorname{dim}_{0} \Sigma f \leq$ $d$, then supp $f$ satisfies the $(d)$-Kouchnirenko condition.

Proof. Put $M=\operatorname{supp} f, M_{i}=\operatorname{supp} f_{z_{i}}^{\prime}, i=1, \ldots, n$. Suppose to the contrary, there exists $I \subset\{1, \ldots, n\}$ such that there are exactly $p<|I|-d$ nonempty sets $M_{j_{1}} \cap O X_{I}, \ldots, M_{j_{p}} \cap O X_{I}$ among the sets $M_{i} \cap O X_{i}, i=1,2, \ldots, n$. Therefore $M_{k} \cap O X_{I}=\emptyset$ for $k \in\{1,2, \ldots n\} \backslash\left\{j_{1}, \ldots, j_{p}\right\}$. For such $k$ we get

$$
\frac{\partial f}{\partial z_{k}}=\sum_{i \notin I} z_{i} h_{i} \quad \text { and } \quad\left\{z \in \mathbb{C}^{n}: z_{i}=0, i \notin I\right\} \subset\left\{\frac{\partial f}{\partial z_{k}}=0\right\}
$$


for some $h_{i} \in \mathcal{O}^{n}$. Substitute $z_{i}=0$ for $i \notin I$ to the system of equations:

$$
\frac{\partial f}{\partial z_{j_{1}}}=\cdots=\frac{\partial f}{\partial z_{j_{p}}}=0 .
$$

We get a system of $p$ equations with $|I|$ variables. Therefore by (1) and Corollary 8 in $[3$, p. 81$]$ we get

$$
\operatorname{dim}\{\nabla f=0\} \geq|I|-p>d
$$

which contradicts the assumption that $\operatorname{dim}_{0} \Sigma f \leq d$.

Remark 4.2. The proof of the above proposition is analogous to the proof "in one side" of the main result in [6] in the case of an isolated singularity. See also Corollary 3.12 in [9].

It turns out that the critical locus of a nondegenerate singularity lies in the sum of the coordinate hyperplanes.

Proposition 4.3. Let $f:\left(\mathbb{C}^{n}, 0\right) \rightarrow(\mathbb{C}, 0), n \geq 2$, be a nonzero nondegenerate singularity. Then $\Sigma f \subset V\left(z_{1} \cdots z_{n}\right)$.

Proof. Suppose to the contrary $\Sigma f \not \subset V\left(z_{1} \cdots z_{n}\right)$. Then by the Curve Selection Lemma there exists a holomorphic parametrization $\varphi:(\mathbb{C}, 0) \rightarrow\left(\mathbb{C}^{n}, 0\right), \varphi_{i} \neq$ $0, i=1, \ldots, n$, such that $(\operatorname{grad} f) \circ \varphi=0$. Now by $[8$, Corollary 2.4.] we get $f$ is degenerate, a contradiction.

Proposition 4.4. Let $f:\left(\mathbb{C}^{n}, 0\right) \rightarrow(\mathbb{C}, 0), n \geq 2$, be a nondegenerate singularity. If $\operatorname{dim}_{0} \Sigma f=n-1$, then supp $f$ does not satisfy the $(n-2)-K o u c h n i r e n k o$ condition.

Proof. By formula $\left(^{*}\right)$ in [7, Section II.5.3] $\Sigma f=V(g) \cup W$, where $g=$ $\operatorname{gcd}\left(f_{z_{1}}^{\prime}, \ldots, f_{z_{n}}^{\prime}\right)$ and $\operatorname{dim} W \leq n-2$. Hence and by Proposition 4.3 we get $V(g) \subset V\left(z_{1} \cdots z_{n}\right)$. Therefore $z_{i} \mid g$ for some $i$. Without loss of generality we may take $i=1$. So $z_{1} \mid f_{z_{i}}^{\prime}, i=1, \ldots, n$. Putting $I=\{1, \ldots, n\} \backslash\{1\}$, we get $\operatorname{supp} f_{z_{i}}^{\prime} \cap O X_{I}=\emptyset, i=1, \ldots, n$. Hence we do not find $|I|-(n-2)=1$ nonempty sets among supp $f_{z_{i}}^{\prime} \cap O X_{I}, i=1, \ldots, n$. Summing up supp $f \operatorname{does}$ not satisfy the $(n-2)$-Kouchnirenko condition.

As a direct corollary of Propositions 4.1 and 4.4 we have the following proposition.

Proposition 4.5. Let $f:\left(\mathbb{C}^{n}, 0\right) \rightarrow(\mathbb{C}, 0)$ be a nondegenerate singularity. The following conditions are equivalent.

(i) $\operatorname{dim}_{0} \Sigma f=n-1$,

(ii) supp $f$ satisfies the $(n-1)$-Kouchnirenko condition and does not satisfy the $(n-2)$-Kouchnirenko condition.

Using Proposition 4.5 we give a simple characterization of a nondegenerate singularity, when its critical locus has codimension one. 
Proposition 4.6. Let $f:\left(\mathbb{C}^{n}, 0\right) \rightarrow(\mathbb{C}, 0)$ be a nondegenerate singularity. The following conditions are equivalent.

(i) $\operatorname{dim}_{0} \Sigma f=n-1$,

(ii) There exists $i \in\{1, \ldots, n\}$ and nonzero $g \in \mathbb{C}\left\{z_{1}, \ldots, z_{n}\right\}$ such that $f=z_{i}^{2} g$.

Proof. If (ii) holds then $V\left(z_{i}\right) \subset \Sigma f$. Hence $\operatorname{dim}_{0} \Sigma f=n-1$. If (i) is true by Proposition 4.5 supp $f$ does not satisfy the $(n-2)$-Kouchnirenko condition. Hence supp $f$ does not satisfy the $(n-2)$-Kouchnirenko condition for some $I,|I| \geq n-1$. Consider the cases.

- $|I|=n$. Since supp $f$ satisfies the $(n-1)$-Kouchnirenko condition then exactly one among the sets $\operatorname{supp} f_{z_{1}}^{\prime}, \ldots, \operatorname{supp} f_{z_{n}}^{\prime}$ is nonempty. Therefore $f$ depends only on $z_{i}$ for some $i$. As $f$ is a singularity $\operatorname{ord}_{z_{i}} f \geq 2$ and we get ii).

- $|I|=n-1$. Then $I=\{1, \ldots, n\} \backslash\{i\}$ for some $i$ and sets

$$
\operatorname{supp} f_{z_{1}}^{\prime} \cap O X_{I}, \ldots, \operatorname{supp} f_{z_{n}}^{\prime} \cap O X_{I}
$$

are empty. Hence $\operatorname{ord}_{z_{i}} f \geq 2$ and ii) holds.

Now, we are ready to prove Theorem 3.2. For a convenience of the reader we will give it again here.

Theorem 3.2. Let $f:\left(\mathbb{C}^{n}, 0\right) \rightarrow(\mathbb{C}, 0), n \leq 3$, be a nondegenerate singularity. The following conditions are equivalent.

(i) $\operatorname{dim}_{0} \Sigma f=d$,

(ii) supp $f$ satisfies the $(d)$-Kouchnirenko condition and does not satisfy the $(d-1)$-Kouchnirenko condition,

$0 \leq d \leq n$.

Proof. Since the conditions (ii) are disjoint for different $d$, it is enough to prove only the implication from (i) to (ii). The case $n=1$ is trivial. Assume that (i) holds and $n>1$. Then by Proposition 4.1 supp $f$ satisfies the $(d)$ Kouchnirenko condition. Now, we show that supp $f$ does not satisfy the $(d-$ 1)-Kouchnirenko condition. Consider the cases:

- $d=n$. Then $f \equiv 0$ and supp $f$ does not satisfy the $(n-1)-$ Kouchnirenko condition.

- $d=n-1$. It follows from Proposition 4.5

- $d=0$. It is easy to check that $\operatorname{supp} f$ does not satisfy the $(-1)-$ Kouchnirenko condition.

It finishes the proof for $n=2$. If $n=3$ and $d=1$ by the main result of [2] we get supp $f$ does not satisfy the Kouchnirenko condition. It finishes the proof for $n=3$.

Example 4.7. Let $f(x, y, z)=z^{3} x+z x^{3}+z y^{3}$. It is a nondegenerate singularity. It is easy to check that supp $f$ satisfy the (1)-Kouchnirenko condition. 
Take $I=\{1,2\}$. Only supp $f_{z}^{\prime} \cap O X_{I} \neq \emptyset$. Hence supp $f$ does not satisfy the Kouchnirenko condition. By the above theorem $\operatorname{dim}_{0} \Sigma f=1$.

We have the following corollary.

Corollary 4.8. Let $f, g:\left(\mathbb{C}^{n}, 0\right) \rightarrow(\mathbb{C}, 0), n \leq 3$, be singularities. If $g$ is a nondegenerate singularity and $\operatorname{supp} f \subset \operatorname{supp} g$ then $\operatorname{dim}_{0} \Sigma g \leq \operatorname{dim}_{0} \Sigma f$.

Proof. Put $d=\operatorname{dim}_{0} \Sigma f$. By Proposition 4.1 supp $f$ satisfy the $(d)$ Kouchnirenko condition. Since supp $f \subset \operatorname{supp} g$ then supp $g$ also satisfies the $(d)$-Kouchnirenko condition. Suppose to the contrary, that $\operatorname{dim}_{0} \Sigma g=$ $d+i, i \geq 1$. Then by the above theorem supp $g$ does not satisfy the $(d+i-1)-$ Kouchnirenko condition. Hence supp $g$ does not satisfy the $(d)$-Kouchnirenko condition, contradiction.

As a direct consequence of the above corollary we get the following.

Corollary 4.9. Let $f, g:\left(\mathbb{C}^{n}, 0\right) \rightarrow(\mathbb{C}, 0), n \leq 3$, be singularities. If $f+g$ is a nondegenerate singularity and $\operatorname{supp} f \cap \operatorname{supp} g=\emptyset$ then

$$
\operatorname{dim}_{0} \Sigma(f+g) \leq \min \left\{\operatorname{dim}_{0} \Sigma f, \operatorname{dim}_{0} \Sigma g\right\} .
$$

Example 4.10. The assumption that $f+g$ is a nondegenerate singularity is necessary in the above corollary. Indeed, take $f(x, y)=x^{2}+y^{2}$ and $g=2 x y$. Then $f+g$ is degenerate and

$$
\operatorname{dim}_{0} \Sigma(f+g)=1>0=\min \left\{\operatorname{dim}_{0} \Sigma f, \operatorname{dim}_{0} \Sigma g\right\} .
$$

Now, we are ready to prove Theorem 3.3. For a convenience of the reader we will give it again here.

Theorem 3.3. Let $f, g:\left(\mathbb{C}^{n}, 0\right) \rightarrow(\mathbb{C}, 0), n \leq 3$, be nondegenerate singularities. If $\Gamma_{+}(f)=\Gamma_{+}(g)$, then $\operatorname{dim}_{0} \Sigma f=\operatorname{dim}_{0} \Sigma g$.

Proof. Assume that $\Gamma_{+}(f)=\Gamma_{+}(g)$. Let $0 \leq d \leq n$. By Theorem 3.2 it is enough to show that supp $f$ does not satisfy the $(d)$-Kouchnirenko condition if and only if supp $g$ does not satisfy the $(d)$-Kouchnirenko condition. Assume that supp $f$ does not satisfy the $(d)$-Kouchnirenko condition and consider the cases:

- $d=n$. It is trivial.

- $d=n-1$. Then $f \equiv 0$ and $g \equiv 0$. Hence $\operatorname{supp} f=\operatorname{supp} g=\emptyset$, which finishes the proof in this case.

- $d=n-2$. Consider the subcases.

- At most one among the sets $\operatorname{supp} f_{z_{1}}^{\prime}, \ldots, \operatorname{supp} f_{z_{n}}^{\prime}$ is nonempty. Since $\Gamma_{+}(f)=\Gamma_{+}(g)$, then $\operatorname{supp} f_{z_{i}}^{\prime}=\emptyset$ if and only if $\operatorname{supp} g_{z_{i}}^{\prime}=\emptyset$. Hence $\operatorname{supp} g$ also does not satisfy the $(n-2)$-Kouchnirenko condition. 
- Then there exists $I,|I|=n-1$, such that all sets

$$
\operatorname{supp} f_{z_{1}}^{\prime} \cap O X_{I}, \ldots, \operatorname{supp} f_{z_{n}}^{\prime} \cap O X_{I}
$$

are empty. Without loss of generality we may assume that $I=$ $\{1, \ldots, n-1\}$. Hence $\operatorname{ord}_{z_{n}} f \geq 2$. Since $\Gamma_{+}(f)=\Gamma_{+}(g)$, we get $\operatorname{ord}_{z_{n}} g \geq 2$. Therefore supp $g$ also does not satisfy the $(n-2)$ Kouchnirenko condition.

It finishes the proof for $n=2$. If $n=3$ and $d=0$, then the assertion follows from [2, Corollary 3.12].

Open Access. This article is licensed under a Creative Commons Attribution 4.0 International License, which permits use, sharing, adaptation, distribution and reproduction in any medium or format, as long as you give appropriate credit to the original author(s) and the source, provide a link to the Creative Commons licence, and indicate if changes were made. The images or other third party material in this article are included in the article's Creative Commons licence, unless indicated otherwise in a credit line to the material. If material is not included in the article's Creative Commons licence and your intended use is not permitted by statutory regulation or exceeds the permitted use, you will need to obtain permission directly from the copyright holder. To view a copy of this licence, visit http://creativecommons.org/ licenses/by/4.0/.

\section{References}

[1] Arnold, V.I.: Arnold's Problems. Springer, Berlin (2004)

[2] Brzostowski, S., Oleksik, G.: On combinatorial criteria for non-degenerate singularities. Kodai Math. J. 39, 455-468 (2016)

[3] Gunning, R.C.: Introduction to Holomorphic Functions of Several Variables. Wadsworth \& Brooks/Cole, vol. II. Springer, Berlin (1990)

[4] Hertling, C., Kurbel, R.: On the classification of quasihomogeneous singularities. J. Singul. 4, 131-153 (2012)

[5] Kouchnirenko, A.G.: Polyèdres de Newton et nombres de Milnor. Invent. Math. 32, 1-31 (1976)

[6] Kouchnirenko, A.G.: Criteria for the existence of a non-degenerate quasihomogeneous function with given weights. Usp. Mat. Nauk. 32, 169-170 (1977). (In Russian)

[7] Łojasiewicz, S.: Introduction to Complex Analytic Geometry. Springer, Basel (1991)

[8] Oleksik, G.: The Łojasiewicz exponent of a nondegenerate surface singularity. Acta. Math. Hung. 138, 179-199 (2013)

[9] Oleksik, G.: On combinatorial criteria for isolated singularities. In: Krasiński, T., Spodzieja, S. (eds.) Analytic and Algebraic Geometry, pp. 81-94. Łódź University Press, Łódź (2013) 
Grzegorz Oleksik

Faculty of Mathematics and Computer Science

University of Lodz

Banacha 22

90-238 Lodz

Poland

e-mail: grzegorz.oleksik@wmii.uni.lodz.pl

Received: December 31, 2019.

Accepted: March 6, 2020.

Publisher's Note Springer Nature remains neutral with regard to jurisdictional claims in published maps and institutional affiliations. 